\title{
Hypothenar Hammer Syndrome: Case Reports and Brief Review
}

\author{
C. Timothy Ablett, MD, MPH and Laurel A. Hackett, FNP, COHN-S
}

\begin{abstract}
Patients with hand and finger pain regularly present to primary care practices. Although a wellknown clinical entity to specialists, hypothenar hammer syndrome is an uncommon vascular overuse syndrome that may not be familiar to primary care practices. It is caused by trauma to the palmar portion of the ulnar artery, usually as a result of occupational or sports activities which involve repetitively striking objects with the heel of the hand. In this report we describe two representative cases as well as discuss clinical features, pathogenesis, diagnosis, imaging and management.
\end{abstract}

Keywords: Allen text; Digital ischemia; Hand injuries; Hypothenar hammer syndrome; Occupational;

Repetitive hypothenar trauma; Ulnar artery occlusion

Reprint Requests:

C. Timothy Ablett, MD, MPH

Occupational Health

Marshfield Clinic

1000 North Oak Avenue

Marshfield, WI 54449

Tel: $715-221-7008$

Fax: 7|5-389-3808

Email: ablett.charles@marshfieldclinic.org

Received: May 10, 2007

Revised: October 4, 2007

Accepted: October 17, 2007

doi: $10.3121 / \mathrm{cmr} .2008 .775$

\begin{abstract}
$\mathrm{P}$ atients regularly present to primary care practices with hand and finger pain. Usually musculoskeletal system disorders are the cause. In occupational and sports medicine practices this problem is often attributed to an overuse type disorder. Again, this most often involves the musculoskeletal system. On a rare basis, however, adults can experience hand and finger pain caused by vascular insufficiency as a result of a variety of vascular overuse syndromes. Case 1 illustrates and discusses an uncommon vascular overuse syndrome, the hypothenar hammer syndrome. Case 2 illustrates and discusses the diagnosis process in a case initially diagnosed as hypothenar hammer syndrome, later confirmed to be systemic lupus erythematosus. Presentation of both cases to the primary care physician refreshes consideration of differential diagnosis.

\section{Case Reports}

Case 1

The patient is a 36-year-old right-hand dominant male who presented to the Occupational Health Department for evaluation of a cold, painful and blanched right fourth digit. The patient is a mechanic by profession. Two days previously he had started his workday by utilizing pliers with his right hand to crimp some wires. This did not require excessive force or repetition but afterwards the patient became aware of coldness and whiteness of his right fourth digit. These symptoms were initially episodic but were persistent 2 days later when he presented for evaluation. He did not report any use of tools with excessive vibration prior to this episode. When queried, he stated that "sometimes" he would use the hypothenar portion of his right hand to force mechanical parts into place. He had no personal or family history of Raynaud's disease, clotting disorders or other vascular disorders. He had no history of tobacco or recreational drug use.
\end{abstract}




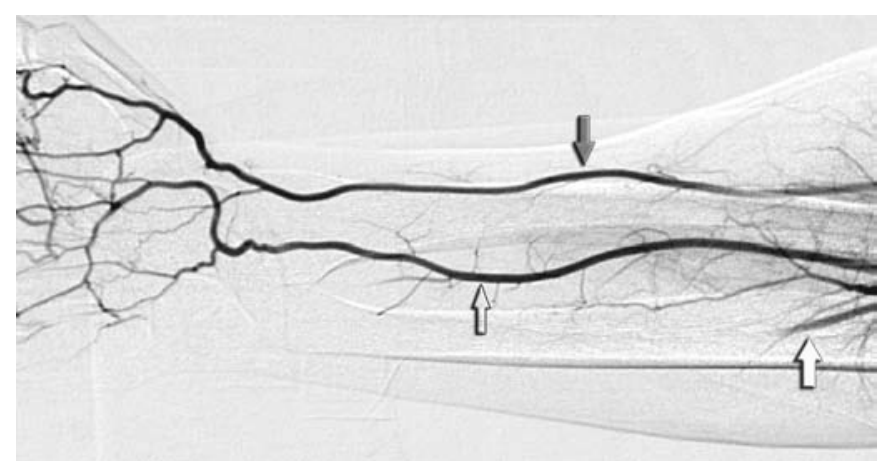

Figure 1. Upper extremity arteriogram showing normal radial artery (dark arrow) and interosseous artery (open arrow). The ulnar artery demonstrates delayed filling (white arrow) compared with the others, suggesting distal stenosis or occlusion.

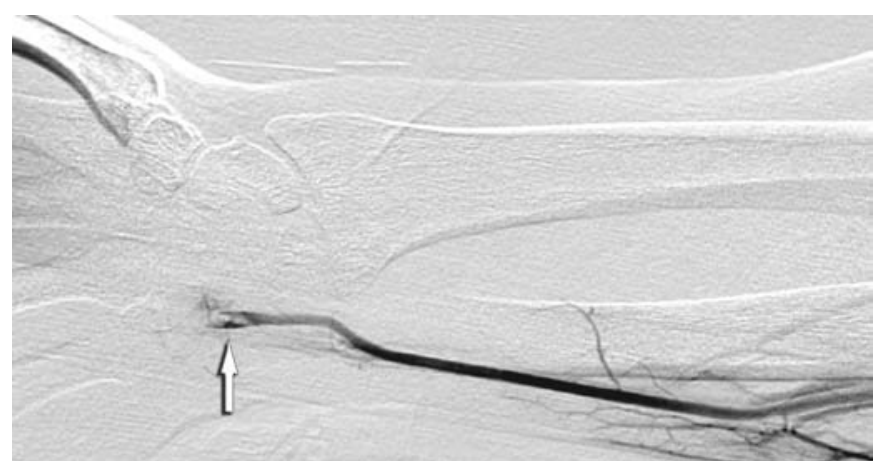

Figure 2. Upper extremity arteriogram showing ulnar artery occlusion (white arrow).
On examination, the patient appeared comfortable. The hands were heavily callused and of normal coloration with the exception of the right fourth digit which was pale and cool to the touch. There was normal range of movement in the fingers and thumbs and normal light touch sensation in the tips of the fingers. Capillary refill was less than 2 seconds in the tips of all fingers except the right fourth where it was 4 seconds. There was a normal Allen test in the left hand with refill of the palmar vasculature in less than 5 seconds. On the right, however, there was normal refill of the palmar vasculature with release of the radial artery but no refill with release of the ulnar artery. The patient's cardiac examination was normal with no murmurs or gallops appreciated.

The patient was referred to vascular surgery and admitted to the hospital for anticoagulation. He was screened for central embolic sources and arterial Doppler studies were suggestive of an ulnar artery occlusion. A right upper extremity angiogram was performed. This demonstrated normal vasculature with the exception of the ulnar artery which was occluded by thrombus at the level of the hamate bone (figures 1 and 2). No aneurysm or pseudoaneurysm was visualized. There was additional occlusion of the medial fourth digital artery and embolus perceived in the lateral fourth digital artery. This was thought to be consistent with a diagnosis of hypothenar hammer syndrome. The patient was discharged on a platelet aggregation inhibitor (clopidogrel) having regained perfusion to his fingers.

On follow-up with a hand surgeon 2 weeks after hospital discharge, the patient complained of mild cold sensitivity in his right fourth digit with distal numbness and paresthesias. On examination he was noted to be tender along the course of the ulnar artery in Guyon's canal and continued to display a positive Allen test with no filling of the digital circulation from the thrombosed ulnar artery. Capillary refill in the right fourth digit continued to be delayed at 5 to 10 seconds compared to the other digits of the right hand. He was advised to avoid further pounding trauma to his hands by utilizing tools such as rubber mallets and to relay this advice to his fellow mechanics. It was recommended that the patient remain on clopidogrel for one additional month and then begin therapeutic aspirin. No further medical treatment was recommended. It was the opinion of the hand surgeon that the complete thrombosis of the ulnar artery and distal embolic disease of the fourth digit would result in permanent but mild impairment of circulation to that digit. The injury was thought by all providers involved to be work-related. Ten months after initial presentation, a follow-up interview indicated that the patient's only remaining symptom was cold sensitivity of his right hand. He was able to perform his usual work without impairment.

\section{Case 2}

The patient is a 23-year-old right-hand dominant male, otherwise in good health, who presented initially to Urgent Care after experiencing several weeks of left third, fourth and fifth finger pain. He described the affected fingertips as being tender and sensitive to touch. He reported that the pain was waking him at night. He had been working but was using his left hand less due to pain. The patient attributed the pain to his work activities as a sheet metal worker. On examination, the patient's left hand showed two small papules at the base of the small fingernail but no other skin changes. Motor and sensory testing was intact but he was hyperesthetic toward pain response at the fingertips as well as the palmar surfaces of the left third, fourth and fifth fingers. The diagnosis at this point was neuropraxia and the patient was placed on antiinflammatory medication.

One week later the patient presented to the Occupational Health Department for re-evaluation. He had been taking ibuprofen with some pain relief. He continued to have moderate pain but it was less tender on the fingertips. He had developed some redness over the tip of the left fifth finger and he felt the left fourth finger was cold. As his activities were reviewed, he stated that he was self-employed as a sheet metal worker but had worked very little over the past few months. He did admit to using his left hand as a hammer at times while working with sheet metal. He had started playing softball 5 weeks prior to this visit, playing several nights a week. He also played basketball on a regular basis. He felt his 
hand problem might be related to catching balls at short-stop or from playing basketball. He denied any pain above the wrist area and denied any paresthesias. He denied use of tobacco.

On examination, there was erythema of the skin over the distal phalanx of the third, fourth and fifth fingers of the left hand. No edema or other skin changes were noted. The fourth finger was cool to the touch as compared to the other fingers. He was tender to palpation over the distal phalanx of the fourth and fifth fingers. He had full range of motion of the fingers and 5/5-muscle strength. Allen testing on the left hand revealed brisk radial flow but greater than 10 seconds of delayed flow from the ulnar artery. The right hand displayed normal Allen testing. The diagnosis at this time was finger pain with vasomotor symptoms of the left hand.

The patient was seen in consultation by a plastic surgeon. It was felt that he had a normal hand examination except for the positive Allen test. Upper extremity arterial Doppler studies demonstrated ulnar artery flow restriction with a probable ulnar artery occlusion. Flow reduction was noted in the fifth finger and, to a lesser extent, in the fourth finger of the left hand. The diagnosis of hypothenar hammer syndrome was made by the hand specialist (plastic surgeon).

An arteriogram of the left upper extremity was performed and the findings were: (1) occluded ulnar artery and superficial palmar arch, likely secondary to trauma, (2) no evidence of reversible vasospasm and (3) occluded digital arteries in the second, fourth and fifth fingers. The patient was advised to avoid further trauma to his palms. He was started on a calcium channel blocker (nifedipine) and consideration was given to resection of the ulnar artery in Guyon's canal.

At his 3-week follow-up with the plastic surgeon, the patient was tolerating the medication well. But, as no improvement was noted, it was recommended he continue on the medication and be seen again in 3 weeks.

At time of follow-up the patient presented instead to Urgent Care for left lower extremity pain and swelling. The patient reported that about 3 weeks earlier he had developed pain in the thigh while playing softball and suspected that he had a muscle strain. Lower extremity venous Doppler studies were performed which demonstrated extensive deep vein thromboses in the common femoral, deep femoral, superior femoral, popliteal, common tibial peroneal trunk, peroneal, posterior tibial and deep muscle vein of the calf. He was admitted to the hospital. During his 9-day hospitalization he developed a pulmonary embolism and required placement of a vena caval filter and catheter-directed thrombolysis in the left lower extremity. He was evaluated for a clotting disorder and found to have antiphospholipid antibody syndrome. It was believed that his initial problems with the left ulnar artery might have been an early sign of hypercoagulability.
The patient will maintain life-long anticoagulation. Follow-up laboratory evaluation demonstrated positive lupus anticoagulant and anticardiolipin antibodies, and the patient was subsequently diagnosed with systemic lupus erythematosus. In retrospect, it was difficult to assign a workrelated causation to the patient's ulnar artery occlusion. The patient was noted as "asymptomatic on hydroxychloroquine" in a follow-up visit 4 years after his initial presentation.

\section{Discussion}

Upper extremity digital ischemia can have a number of causes including Raynaud's disease, Raynaud's phenomenon associated with connective tissue disorders, vasculitis, arterial emboli from a cardiac source, thromboangiitis obliterans, atherosclerosis with secondary thrombosis and thoracic outlet syndrome. ${ }^{1,2}$ To this differential diagnosis can be added a work-related and less common cause of digital ischemia, the hypothenar hammer syndrome, as exemplified in the case reports described above. This clinical entity was first described by Guttani ${ }^{3}$ and Von Rosen. ${ }^{4}$ The term "hypothenar hammer syndrome" was suggested by Conn et al ${ }^{5}$ in 1970 who proposed that repeated blunt trauma to the hypothenar portion of the hand can lead to ulnar artery damage by the mechanism described below.

Typically, hypothenar hammer syndrome occurs in men with a mean age of 40 years $^{6}$ involving the dominant hand and in occupational settings where the worker uses the hypothenar portion of the hand as a tool to hammer, push or squeeze hard objects. Workers most at risk include metal workers, auto mechanics, lathe operators, machinists, miners, sawmill workers, butchers, bakers, brick layers and carpenters.

Although generally described as a rare condition, hypothenar hammer syndrome may occur more frequently than acknowledged. ${ }^{7}$ In one series of auto mechanics who gave a history of habitual occupational hammering, 14\% showed clinical and Doppler evidence of hypothenar hammer syndrome $^{8}$ which can also be associated with another occupational diagnosis, hand vibration syndrome. This is a complex syndrome involving vessels, nerves, muscles and joints, which occurs with the prolonged use of air hammers, chain saws, impact wrenches and other power tools that generate vibration. Hypothenar hammer syndrome was found in $7 \%$ of one group of 330 vibration-exposed workers. ${ }^{9}$ It can occur occasionally in athletes who experience trauma to the palm and has been reported in baseball, karate, badminton, mountain biking, golf, tennis, handball, volleyball, softball, weight lifting, break-dancing and hockey. Occasionally, a single episode of significant trauma can be the cause of hypothenar hammer syndrome. ${ }^{10}$ It is not uncommon for the initiating injury to seem trivial and consequently ignored with resulting misdiagnosis and disability. ${ }^{11}$

\section{Anatomy/Pathogenesis}

The pathogenesis of the hypothenar hammer syndrome is related to the anatomy of the ulnar artery as it enters the palm. 
At Guyon's canal, the ulnar artery branches off to the deep palmar arch and exits the canal to form the superficial palmar arch. This superficial branch of the ulnar artery crosses the surface of the hypothenar muscles for about $2 \mathrm{~cm}$ before penetrating the palmar aponeurosis. This arterial segment is very susceptible to injury as it is superficial with only limited protection from scant overlying tissues. With palmar trauma, this vascular segment can be compressed against the adjacent boney hook of the hamate (also known as the hamulus), much like an object being fashioned with a hammer on an anvil.

The superficial palmar branch of the ulnar artery provides the main source of blood for most of the fingers. The superficial palmar arch is completed on the radial side in a variable way, by junction with the radialis indicis or the princeps pollicis artery. This communication is often small, measuring less than $2 \mathrm{~mm}$ in diameter. In $37 \%$ of anatomic cases, the superficial arch is believed to arise entirely from the ulnar artery. In $16 \%$ to $22 \%$ of patients, the superficial arch is incomplete. ${ }^{12,13}$

With repetitive blunt trauma, the superficial palmar branch of the ulnar artery can develop intimal damage producing vasospasm and encouraging platelet aggregation and thrombus formation. If the damage spreads through the media into the arterial wall, aneurysm formation can occur as well. The periadventitial tissues can become involved, leading to extrinsic scarring, vascular narrowing and thrombosis. Microemboli can be generated that can occlude the digital arteries downstream, resulting in digital ischemia. It is hypothesized that a thrombosed artery segment may act as a disease plexus, initiating reflexes that lead to peripheral vasospasm. In the hand, these reflexes occur at the second, third and fourth thoracic segments. ${ }^{14}$ Neurologic symptoms such as paresthesias and pain may also result from compression of the sensory branches of the ulnar nerve which run in close proximity to the ulnar artery.

Because of the variable anatomy of the palmar circulation as described above, the effects of these processes can be highly variable from no symptoms to digital gangrene in injured workers and recreationists. Given the universality of use of the hands for hammering in the workplace, it is noteworthy that hypothenar hammer syndrome is a rare clinical problem. Blum et al ${ }^{15}$ point out that the position of the ulnar artery relative to the hamulus (which is quite variable) and the site of the impact over the hypothenar eminence may determine the risk of developing an ulnar artery lesion.

A second explanation for this disparity is suggested by Ferris et al ${ }^{16}$ who reported a large series (21 male cases) of hypothenar hammer syndrome and found that all had repetitive palmar trauma. In addition, they found a high incidence of bilateral ulnar artery abnormalities in patients with unilateral symptomatology. These arteriographic abnormalities consisted of a "corkscrew" configuration to the ulnar artery, representing alternating areas of stenosis and ectasia. Histologic evaluation of this group's postoperative specimens showed pathology suggestive of the syndrome of fibromuscular dysplasia. This is an angiopathy that affects medium-sized arteries, predominantly in young women of child-bearing age. The authors proposed that patients with hypothenar hammer syndrome have intrinsically abnormal arteries, predisposing them to injury from palmar trauma. The authors admit, however, that this hypothesis does not explain the preponderance of hypothenar hammer syndrome in male patients, who do not show the renal and cerebrovascular disease typical of fibromuscular dysplasia in others. Another interesting pathologic observation is offered by Stone. ${ }^{17} \mathrm{He}$ analyzed random autopsy specimens of distal ulnar arteries and found intimal hyperplasia to be a common finding in this injury-prone vascular segment. Additionally, he found that the degree of intimal hyperplasia was greater in the right hands of men than in either their corresponding left hands or in women. He speculated that these findings were a consequence of repetitive trauma and did not represent an angiopathy, such as fibromuscular dysplasia.

\section{Presentation}

Arterial damage may present dramatically or with subtle, intermittent signs and symptoms that may be temporally remote from the episode of injury. ${ }^{18}$ Typically, a male patient will present with ischemia of the second, third, fourth or fifth digits of the dominant hand. Patients may complain of digital pain with paresthesias, cold sensitivity, phasic blanching or discoloration of the fingertips, finger claudication or hypothenar pain. Occasionally, ischemic symptoms are present but not of sufficient severity for the patient to seek medical evaluation.

\section{Examination}

On physical examination, hypothenar callus and tenderness may be present in the dominant hand. A pulsatile hypothenar mass may be present in the case of aneurysm formation. There may be episodic blanching or purplish discoloration of the second, third, fourth or fifth fingertips. These changes lack the erythematous blush phase of the classic triphasic white/blue/red changes of Raynaud's phenomenon. In addition, these findings are usually unilateral in contrast to the bilateral findings in connective tissues disorders, Raynaud's disease and thromboangiitis obliterans. ${ }^{10}$ Pulp wasting, splinter hemorrhages and coldness may be present in the affected fingertips. In severe cases, gangrene, ulceration or eschar formation of the involved fingertips may occur.

\section{Diagnostic Testing}

An Allen test, which assesses the patency of the superficial palmar arch, can provide important diagnostic clues. This test is performed with the patient sitting with the hands supinated on the knees. The examiner compresses the radial artery with one thumb and the ulnar artery with the other thumb. The patient exsanguinates the hand by rapidly clenching and opening the fist several times (care must be taken to not over-extend the fingers which can lead to a false-positive 
result). The ulnar artery is released and the palm is observed for the return of normal color. This should occur within 5 seconds. If the ulnar artery is occluded, palmar pallor will be maintained until the radial artery is released. A positive Allen test suggests occlusion, stenosis or incomplete development of the superficial palmar arch or distal ulnar artery. In one series, however, the Allen test was negative in $14 \%$ of patients with hypothenar hammer syndrome. ${ }^{9}$ Others have observed that the Allen test is lacking in specificity with positive findings in $22 \%$ of normal patients. ${ }^{19}$ Noninvasive testing that can be helpful in establishing the diagnosis of hypothenar hammer syndrome includes thermography, cold stress testing, digital pulse volume recordings and plethysmography.

More often, Doppler color pencil mapping is used to assess blood flow and site of thrombus formation in the ulnar artery and the superficial palmar arch. This test is convenient but requires operator experience to carefully map the arterial tree and the patient must avoid movement during the entire examination. The benefit of Doppler mapping is that it does not induce vasospasm and may provide a demonstration of arterial hemodynamics.

The "gold standard" for establishing the diagnosis of hypothenar hammer syndrome is angiography. An aortic arch and upper extremity arteriogram provides the most information. According to Vayssairat et al, ${ }^{6}$ this includes: (1) locate vascular occlusion (subclavian versus distal), (2) confirm the exact location of vascular damage to the end of the ulnar artery, (3) define the nature of the ulnar artery disturbance (vasospasm versus thrombus versus aneurysm) and (4) evaluate the presence of digital artery emboli. Additionally, angiography defines the anatomy of the palmar arch and other information necessary to plan surgical intervention. ${ }^{20}$

The retrograde femoral Seldinger technique of upper extremity arteriography is preferred by several authors. ${ }^{6,10}$ This technique allows for better visualization of the subclavian artery and aortic arch. It is also safer in the unlikely event of an arteriographic accident in an already compromised upper extremity circulation.

In hypothenar hammer syndrome, the pathognomonic angiographic features can include: (1) tortuosity of the ulnar artery with a typical "corkscrew" appearance, (2) aneurysm formation, (3) occlusion of the ulnar artery segment overlying the hook of the hamate, (4) occluded digital arteries in an ulnar artery distribution, and (5) demonstration of intraluminal emboli at sites of digital obstruction.

Blum et al $^{15}$ state that an alternative to conventional arteriography for evaluating hypothenar hammer syndrome is multi-detector computed tomography angiography. The authors point out that this technique not only displays pertinent vascular anatomy but can rule out bone abnormalities such as hamate fracture, and can show the relationship of the ulnar artery to the hamulus. Magnetic resonance image angiography has also been utilized for evaluation of ulnar artery trauma and can demonstrate accessory muscles or ossicles near the ulnar artery that increase the risk for traumatic lesions. ${ }^{21}$

\section{Treatment}

The therapeutic strategy for treatment of hypothenar hammer syndrome is controversial due to limited studies on this rare problem. ${ }^{22}$ For most patients non-surgical treatment will be sufficient, particularly in the setting of vasospasm with adequate collateral circulation. Conservative non-operative care may include: (1) smoking cessation, (2) avoidance of further trauma (may require change of occupation), (3) padded protective gloves, (4) cold avoidance, (5) calcium channel blockers (nifedipine, diltiazem), (6) antiplatelet agents or anticoagulation, (7) local care of fingers with necrosis, and (8) pentoxifylline to reduce blood viscosity.

Often decisions regarding surgical versus non-surgical treatments are based on the acuteness of the symptoms and severity of the ischemia. Angiography may demonstrate more significant vascular damage, poor collateral circulation and/or vasospasm. Surgical options in this setting include: (1) arterial ligation (assuming an intact radial/palmar arch), (2) resection of thrombosed arterial segment or aneurysm with end-to-end anastomosis, and (3) resection and vascular reconstruction with vein or artery graft.

Although based on small numbers of patients, some argue that best outcomes are seen in those treated with surgical resection and reconstruction. Vayssairat et al ${ }^{6}$ summarize the advantages of surgical treatment as: (1) removal of a source of embolism, (2) removal of a painful mass, (3) relief of ulnar nerve compression, and (4) creation of a local periarterial sympathectomy.

Overall, however, studies regarding surgical treatment of hypothenar hammer syndrome are limited by small numbers, retrospective study design, variable mixes of pathology (thrombosis versus aneurysm) and treatment modalities (ligation versus vein graft versus arterial graft). ${ }^{23,24}$ Additionally, these procedures are occasionally combined with other treatment modalities such as endoscopic thoracic sympathectomy, ${ }^{14}$ intra-arterial vasodilators such as nitroglycerin and/or intra-arterial thrombolysis with agents such as tissue plasminogen activator or urokinase. ${ }^{25,26}$

Surgeons will argue that recovery of function and return to work is more likely with early, aggressive and definitive treatment. However, as Van de Walle et $\mathrm{al}^{22}$ points out, "Because of the absence in the literature of large series with long term follow up and in light of good results reported for most treatment modalities, one can conclude that different therapeutic options are acceptable for one and the same patient." 


\section{Conclusions}

As described above, hypothenar hammer syndrome may not be as rare as once thought. ${ }^{27}$ It is a curable and preventable cause of upper extremity digital ischemia. Primary care physicians may not be familiar with this etiology of hand and finger symptomatology. ${ }^{28}$ Consequently, hypothenar hammer syndrome is often incorrectly diagnosed. ${ }^{29}$ Most patients with vascular ischemia involving the digits will have an underlying medical illness that precipitates or exacerbates their condition. When that is not the case, however, a careful history for occupational or recreational trauma may point towards a vascular overuse syndrome, such as hypothenar hammer syndrome. Early referral to a hand specialist should be considered as persistent signs and symptoms leading to significant disability are not uncommon with a delayed diagnosis of hypothenar hammer syndrome, noncompliance or insufficient treatment. Since some ulnar arterial damage can go undetected at a preclinical stage, regular screening of hands by occupational health services in involved industries should be considered.

\section{Acknowledgments}

We thank Drs. Timothy Swan and Steven Kirkhorn for their critical review of our article and Marshfield Clinic Research Foundation for its support through the assistance of Anne Nikolai, Alice Stargardt and Linda Weis in the preparation of this manuscript. We are grateful to Dr. Swan for providing the images and Eric Borreson for preparing them for publication.

\section{References}

1. Coffman JD. Vasospastic diseases. In:Young JR, Graor RA, Olin JW, Bartholomew JR, eds. Peripheral vascular diseases. St. Louis, MO: Mosby-Year Book; 1991. 361-377.

2. Rooke TW, Stanson AW. Vascular diseases of the upper limb. In: Young JR, Graor RA, Olin JW, Bartholomew JR, eds. Peripheral vascular diseases. St. Louis, MO: Mosby-Year Book; 1991. 651-667.

3. Guttani C. De externis aneurysmatibus manu chirurgica methodice pertractandis. 1772. In: Erichsen JE, trans. Observations on aneurysm. London: Sydenham Society; 1884. 316-318.

4. Von Rosen S. Ein Fall Von Thrombose in der Arteria Ulnaris nach Einwirkung von Stumper Gewalt. ACTA Chir Scand 1934;73:500-506.

5. Conn J Jr, Bergan JJ, Bell JL. Hand ischemia: hypothenar hammer syndrome. Proc Inst Med Chic 1970;28:83.

6. Vayssairat M, Debure C, Cormier JM, Bruneval P, Laurian C, Juillet Y. Hypothenar hammer syndrome: seventeen cases with long-term follow-up. J Vasc Surg 1987;5:838-843.

7. Gaylis H, Kushlick AR. The hypothenar hammer syndrome. S Afr Med J 1976;50:125-127.

8. Little JM, Ferguson DA. The incidence of the hypothenar hammer syndrome. Arch Surg 1972;105:684-685.

9. Kaji H, Honma H, Usui M, Yasuno Y, Saito K. Hypothenar hammer syndrome in workers occupationally exposed to vibrating tools. J Hand Surg [Br] 1993;18:761-766.

10. Pineda CJ, Weisman MH, Bookstein JJ, Saltzstein SL. Hypothenar hammer syndrome. Form of reversible Raynaud's phenomenon. Am J Med 1985;79:561-570.

11. Duncan WC. Hypothenar hammer syndrome: an uncommon cause of digital ischemia. J Am Acad Dermatol 1996;34:880883.
12. Al-Turk M, Metcalf WK. A study of the superficial palmar arteries using the Doppler Ultrasonic Flowmeter. J Anat 1984;138:27-32.

13. Coleman SS, Anson BJ. Arterial patterns in the hand based upon a study of 650 specimens. Surg Gynecol Obstet 1961;113:409-424.

14. Benedict KT Jr, Chang W, McCready FJ. The hypothenar hammer syndrome. Radiology 1974;111:57-60.

15. Blum AG, Zabel JP, Kohlmann R, Batch T, Barbara K, Zhu X, Dautel G, Dap F. Pathologic conditions of the hypothenar eminence: evaluation with multidetector CT and MR imaging. Radiographics 2006;26:1021-1044.

16. Ferris BL, Taylor LM Jr, Oyama K, McLafferty RB, Edwards JM, Moneta GL, Porter JM. Hypothenar hammer syndrome: proposed etiology. J Vasc Surg 2000;31:104-113.

17. Stone JR. Intimal hyperplasia in the distal ulnar artery; influence of gender and implications for the hypothenar hammer syndrome. Cardiovasc Pathol 2004;13:20-25.

18. Williams DS. Hypothenar hammer syndrome. J Insur Med 2006;38:233-235.

19. Mousavi SM Aneurysms and arterial thrombosis of the hand. Vasc Surg 1978;12:294-305.

20. Karimi KM, Perlmutter AE, Freeman BG. Overuse-related vascular injury of the hand-hypothenar hammer syndrome: a case report. W V Med J 2005;101:250-252.

21. Drape JL, Feydy A, Guerini H, Desmarais E, Godefroy D, Le Viet D, Chevrot A. Vascular lesions of the hand. Eur J Radiol 2005;56:331-343.

22. Van de Walle PM, Moll FL, De Smet AA. The hypothenar hammer syndrome: update and literature review. Acta Chir Belg 1998;98:116-119

23. Dethmers RS, Houpt P. Surgical management of hypotherar and thenar hammer syndromes: a retrospective study of 31 instances in 28 patients. J Hand Surg [Br] 2005;30:419-423.

24. De Monaco D, Fritsche E, Rigoni G, Schlunke S, Von Wartburg U. Hypothenar hammer syndrome. Retrospective study of nine cases. J Hand Surg [Br] 1999;24:731-734.

25. Bakhach J, Chahidi N, Conde A. Hypothenar hammer syndrome: management of distal embolization by intraarterial fibrinolytics. Chir Main 1998;17:215-220.

26. Yakubov SJ, Nappi JF, Candela RJ, George BS. Successful prolonged local infusion of urokinase for the hypothenar hammer syndrome. Cathet Cardiovasc Diagn 1993;29: 301-303.

27. Abudakka M, Pillai A, Al-Khaffaf H. Hypothenar Hammer Syndrome: rare or underdiagnosed? Eur J Vasc Endovasc Surg 2006;32:257-260

28. Wernick R, Smith DL. Bilateral hypothenar hammer syndrome: an unusual and preventable cause of digital ischemia. Am J Emerg Med 1989; 7:302-306.

29. Liskutin J, Dorffner R, Resinger M, Silberbauer K, Mostbeck G. Hypothenar hammer syndrome. Eur Radiol 2000;10:542.

Author Affiliations

C. Timothy Ablett, $M D, M P H$

Occupational Health

Marshfield Clinic

1000 North Oak Avenue

Marshfield, Wisconsin 54449

Laurel A. Hackett, FNP, COHN-S

Occupational Health

Marshfield Clinic

1000 North Oak Avenue

Marshfield, Wisconsin 54449 